\title{
Control de Sitophilus zeamais con polvos vegetales de tres especies del género Chenopodium
}

\author{
Gonzalo Ivan Silva-Aguayo(1), Rodrigo Kiger-Melivilu(1), Ruperto Hepp-Gallo(1) y Maritza Tapia-Vargas ${ }^{(1)}$
}

(1)Universidad de Concepción, Facultad de Agronomía, Dep. de Producción Vegetal, Vicente Méndez № 595, Casilla 537 Chillán, Chile. E-mail: gosilva@udec.cl, rkiger@udec.cl, rhepp@udec.cl, martapia@udec.cl

\begin{abstract}
Resumen - Se evaluaron polvos vegetales de Chenopodium ambrosioides L., Chenopodium album L. y Chenopodium quinoa Willd. para el control de Sitophilus zeamais Motschulsky, bajo condiciones de laboratorio. Los parámetros evaluados fueron mortalidad y emergencia de insectos adultos, pérdida de peso y germinación de los granos, efecto ovicida y larvicida, fumigación, repelencia y residualidad de los polvos. El diseño experimental fue completamente al azar, con un arreglo factorial y tres repeticiones. La mayor mortalidad de insectos se obtuvo con los polvos de la inflorescencia y la mezcla de hojas y tallos de Chenopodium ambrosioides L., al 2\% (p/p) con valores de 69,4\% y 67,9\% respectivamente. La menor emergencia de adultos se obtuvo con los mismos tratamientos. La pérdida de peso de granos, en todos los tratamientos de C. ambrosioides, no superó el 3\%. Para el tratamiento inflorescencia de C. ambrosioides al 2\% (p/p), la residualidad de los polvos se mantuvo hasta los 15 días, con una mortalidad de 98,3\%. Esta misma especie presentó una mortalidad de huevos y larvas de 100\%, además de presentar un efecto fumigante con una mortalidad de adultos de 100\%, en todos los tratamientos evaluados. El polvo de C. ambrosioides es repelente para S. zeamais.
\end{abstract}

Términos para indexación: granos almacenados, insecticidas vegetales, paico, quinoa.

\section{Control of Sitophilus zeamais with vegetable powders of three species of Chenopodium genus}

\begin{abstract}
Powders of Chenopodium ambrosioides L., Chenopodium album L. and Chenopodium quinoa Willd. were evaluated under laboratory conditions for Sitophilus zeamais Motschulsky control. Evaluated parameters were insect mortality and adult emergence, grain weight loss, grain germination and the following effects of powders: ovicide and larvicide, fumigant, repellent and residual. A completely randomized experimental design with factorial arrangement and three replicates was used. The highest levels of insect mortality were obtained with the powders of Chenopodium ambrosioides L. inflorescence and the mixture of leaves and stems at $2 \%(\mathrm{w} / \mathrm{w})$, with values of $69.4 \%$ and $67.9 \%$ respectively. Best emergence reductions were obtained with the same treatments. In all treatments with powders of $C$. ambrosioides seeds weight were reduced in less than 3\%. The residual effect lasted for 15 days, obtaining a mortality of $98.3 \%$ of adult insects, when powders of C. ambrosioides inflorescence were used at $2 \%(\mathrm{w} / \mathrm{w})$ concentration. Powders of C. ambrosioides showed a mortality of $100 \%$ of eggs and larvae, and a fumigation effect against adult insects with a mortality of $100 \%$ in all treatments was evaluated. Powders of $C$. ambrosioides have a repellent effect for S. zeamais.
\end{abstract}

Index terms: stored grains, botanical insecticides, mexican tea, quinoa.

\section{Introducción}

Los cereales son considerados, mundialmente, como las especies vegetales más importantes para la alimentación de los seres humanos y animales domésticos (Dell’Orto \& Arias, 1983). Por esto, su almacenamiento por largos períodos de tiempo es esencial para disponer de alimento en forma constante (Paez, 1987). Lamentablemente, esto se ve entorpecido por los insectos plagas de los granos almacenados, que causan cuantiosas pérdidas, tanto en lo económico como en su disponibilidad para la alimentación de animales y seres humanos (Larraín, 1994). Esto se magnifica en el caso de pequeños agricultores, ya que no cuentan con la suficiente información y tecnología, para realizar un manejo de postcosecha que minimice el daño causado por estas plagas (Paez, 1987). 
Los métodos de control de plagas en granos almacenados son de muy variada naturaleza. Existen desde técnicas altamente sencillas, como el uso de temperaturas extremas y otros métodos físicos como inertes y envases herméticos, hasta el uso de insecticidas sintéticos y atmósfera controlada (Celis \& Kunadu, 1992). Lamentablemente, muchas de estas técnicas no están al alcance de los pequeños agricultores, ya sea por costo o por el riesgo que pueda implicar el uso inadecuado de ellos. En vista de esto, muchas veces los agricultores recurren a elementos disponibles en su medio (Golob \& Hanks, 1990).

La revalorización de las plantas, como fuente de sustancias con propiedades insecticidas, se viene difundiendo desde los últimos 35 años. En algunos países de América Latina se han desarrollado interesantes líneas de investigación, que buscan en las plantas compuestos químicos con menor impacto ambiental y con potencial para el control de plagas agrícolas (Mareggiani, 2001; Mazzonetto, 2002; Tavares, 2002).

El uso más sencillo de las plantas, para el control de plagas de los granos almacenados, es la mezcla física de los polvos secos de estas con el grano (Weaver \& Subramanyan, 2000). Se ha evaluado una gran cantidad de polvos de origen vegetal, para el control de estos insectos en países como Brasil, México y Chile, aunque sus propiedades protectoras son solamente preventivas, ya que una vez que el insecto penetre el grano, el polvo no tendrá efecto (Silva et al., 2001).

El Paico (Chenopodium ambrosioides L.; Chenopodiaceae) ha sido una de las especies vegetales evaluada por algunos autores, quienes han encontrado que posee propiedades insecticidas sobre diversas especies asociadas a granos almacenados (Paez, 1987; Rodríguez \& Lagunes, 1992; Rodríguez, 2000; Mazzonetto, 2002; Tavares, 2002; Procópio et al., 2003a, 2003b; Silva et al., 2003; Orrego, 2004). Las especies Chenopodium album L. (Quingüilla) y Chenopodium quinoa Willd. (Quinoa) pertenecen a la misma familia y género que C. ambrosioides, por lo que sería lógico suponer que estas plantas pudieran compartir algunos de los compuestos químicos, presentes en el Paico, causantes de las propiedades insecticidas reportadas.

De esta forma, el uso de polvos vegetales de especies del género Chenopodium para el control del gorgojo del maíz (S. zeamais Mots.) viene a satisfacer una creciente demanda por investigaciones de este tipo, que tienen la finalidad de obtener productos más inocuos para el ser humano, naturales, de fácil acceso, económicos y efectivamente aplicables, que den una solución a los problemas de los insectos en los granos almacenados (Mazzonetto, 2002; Tavares, 2002).

El objetivo de la presente investigación fue evaluar las propiedades insecticidas/insectistáticas de los polvos de Paico (C. ambrosioides L.), Quingüilla (C. album L.) y Quinoa (C. quinoa Willd.), de modo que constituyan una alternativa natural, de bajo costo, fácil acceso y factible de llevar a cabo, para el control del gorgojo del maíz (S. zeamais Mots.) por pequeños agricultores.

\section{Material y Métodos}

La recolección del material vegetal se realizó en la etapa de floración, ya que en este estado fenológico las plantas contienen la mayor concentración de compuestos (Mazzonetto, 2002; Tavares, 2002). Posteriormente, todo el material recolectado fue secado en una estufa, a una temperatura de $40^{\circ} \mathrm{C}$ por 48 horas, para después ser pulverizado con un molino de café.

Los tratamientos se evaluaron en concentraciones de $0,5 \%, 1 \%$ y $2 \%$ (p/p) en 100 g de maíz cv. Laredo, más un testigo sin polvo vegetal. Se evaluaron en total 21 tratamientos, en un arreglo factorial de $7 \times 3$ (7 tratamientos y 3 concentraciones), y cada tratamiento tuvo tres repeticiones, con el grupo completo de tratamientos tres veces en el tiempo, para disminuir el error experimental.

El uso de maíz se debe a que el tamaño del grano es mayor al del trigo o arroz, lo que facilitó la diferenciación entre los granos dañados por los insectos y los granos sin daño.

Se utilizaron ejemplares adultos de $S$. zeamais, el cual fue reproducido bajo condiciones controladas $\left(30 \pm 2^{\circ} \mathrm{C}\right.$ $\mathrm{y}$ fotofase de 16 horas). Los insectos utilizados en los bioensayos no tenían más de 10 días de edad, para lo cual, 10 días antes de comenzar la experiencia, se eliminaron todos los insectos adultos de las colonias y los que emergieron después de ese período fueron los que se usaron.

La metodología que se utilizó fue la propuesta por Lagunes \& Rodríguez (1989), la cual se realizó con la mezcla en frascos de vidrio de $400 \mathrm{~mL}$ de 100 g de maíz con los diferentes tratamientos a sus respectivas concentraciones de polvo vegetal. Una vez realizada la mezcla, se procedió a infestar cada frasco con 20 parejas de insectos, los cuales fueron diferenciados por sexo, 
de acuerdo a los criterios propuestos por Halstead (1963), quien señala que el rostrum del macho es de menor longitud y más rugoso que el de la hembra. Posteriormente, los frascos fueron colocados dentro de una cámara de cría, con las condiciones de temperatura y fotofase señaladas anteriormente.

Para estimar la toxicidad de cada tratamiento se midieron las siguientes variables: mortalidad y emergencia de adultos y pérdida de peso del grano. La mortalidad se evaluó a los 15 días de realizada la infestación, y se cuantificaron los insectos adultos vivos y muertos de cada tratamiento y sus repeticiones. Luego del conteo, los frascos fueron devueltos a la cámara de cría sin los insectos, pero incorporando nuevamente los polvos vegetales. La valoración del porcentaje de mortalidad se obtuvo utilizándose la fórmula de Abbott (Abbott, 1925).

La emergencia de los adultos fue evaluada 55 días después de la infestación, la que se realizó en todos los tratamientos, habiéndose registrado su valor con respecto al testigo, cuya emergencia se consideró como 100\%. El porcentaje de pérdida de peso de los granos también fue cuantificado a los 55 días a partir de la infestación, habiéndose contabilizado el número de granos sanos y dañados. Para su estimación se utilizó la fórmula propuesta por Adams \& Schulten (1976): $\mathrm{P}_{\mathrm{p}}=100(\mathrm{Ngd} /$ $\mathrm{Ntg}) \mathrm{xC}$ donde Pp es la pérdida de peso (\%); Ngd es el número de granos dañados; Ntg es el número total de granos; C es 0,125, si el maíz es almacenado como grano suelto o mazorca sin brácteas y 0,222 , si el maíz es almacenado como mazorca con brácteas.

La metodología utilizada para la evaluación de repelencia fue adaptada de la propuesta por Mazzonetto (2002) y Tavares (2002). Se utilizó una arena formada por cinco cajas plásticas circulares, de $5 \mathrm{~cm}$ de diámetro y 1,5 cm de altura; la caja central fue conectada con las demás cajas por tubos plásticos de $10 \mathrm{~cm}$ de longitud dispuestos diagonalmente. Las placas con polvo y los testigos, sin polvo vegetal, fueron distribuidos en dos cajas simétricamente opuestas. En el recipiente central se liberaron 50 adultos de $S$. zeamais sin sexar y luego de 24 horas se contabilizó el número de insectos en cada recipiente. Cada tratamiento tuvo cinco repeticiones y se utilizó la formula descrita por Mazzonetto (2002), $\mathrm{IR}=2 \mathrm{G} /(\mathrm{G}+\mathrm{P})$, para determinar el indice de repelencia, donde IR es el índice de repelencia; $G$ es el porcentaje de insectos en el tratamiento y $\mathrm{P}$ es el porcentaje de insectos en el testigo. El polvo vegetal es neutro si IR=1, atrayente si IR $>1$ y repelente si IR $<1$.
La prueba para verificar su efecto ovicida o larvicida se realizó en aquellos tratamientos que obtuvieron una mortalidad superior al $40 \%$, para lo cual se colocaron 100 g de maíz en frascos de $400 \mathrm{~mL}$, los que se infestaron con 20 parejas de insectos que se dejaron copular por 15 días. Al término de este período, todos los insectos fueron retirados y el grano fue mezclado con los polvos vegetales, según el procedimiento antes mencionado, para luego devolver los frascos a la cámara de cría. La emergencia de la progenie se contabilizó a los 55 días de haber sido realizada la infestación.

El efecto fumigante también se evaluó solamente para los tratamientos que mostraron porcentajes de mortalidad sobre un $40 \%$. La metodología se adaptó de la propuesta por Tavares (2002). En frascos de plástico de $200 \mathrm{~mL}$ de capacidad, se dispuso en el fondo y centrado un tubo de PVC, en cuyo interior se colocó polvo de Paico, a las concentraciones de $1 \%, 2 \%$ y $4 \%(\mathrm{p} / \mathrm{p})$, el que fue tapado con un género de tul fino. Por fuera de este tubo se colocó 50 g de maíz cv. Laredo, infestándose cada tratamiento con 20 insectos sin sexar. El género de tul fino impidió el contacto de los insectos con los polvos, pero permitió que se desprendieran al medio los semioquímicos presentes en ellos. La medición se realizó a los cinco días de haber sido realizada la infestación, contabilizándose los insectos adultos vivos y muertos. Cada tratamiento tuvo tres repeticiones, sumándose un testigo igual a los tratamientos pero sin polvo vegetal.

Al igual que en las variables anteriores, en los tratamientos que obtuvieron porcentajes de mortalidad sobre un $40 \%$ se evaluó el efecto residual. Para esto, se repitió la aplicación de los polvos en 1,2kg de maíz, que posteriormente se dividió en un total de 12 submuestras de 100 g cada una. Estas a su vez se dividieron en cuatro grupos de tres submuestras, que fueron infestadas con 10 parejas de insectos, a las 24 horas, 15, 30 y 45 días, constituyendo un arreglo factorial de $4 \times 4$ (4 tratamientos x 4 fechas). Los parámetros evaluados fueron: porcentaje de mortalidad, emergencia de insectos adultos y pérdida de peso de los granos. Además, se realizó un análisis de regresión para mortalidad y emergencia de insectos, para comprobarse analíticamente las tendencias mostradas por ambas variables en el tiempo.

El diseño experimental fue completamente al azar. Los datos que se obtuvieron fueron sometidos a un análisis de varianza utilizándose el software Statistical Analysis System (SAS) versión 8.0 y se realizó una 
comparación de medias de los tratamientos, mediante una prueba de Tukey, a un nivel de significancia del 5\%. Para el análisis estadístico, todos los datos obtenidos fueron transformados en arc sen $(\mathrm{x} / 100)^{0,5}$.

\section{Resultados y Discusión}

Considerándose el criterio propuesto por Silva et al. (2003), quienes señalan como prometedores sólo aquellos tratamientos con una mortalidad superior al $40 \%$, se puede indicar que de los 21 polvos vegetales evaluados sólo tres sobrepasaron el umbral fijado.

El análisis demuestra que existe interacción entre la concentración y el tratamiento. Los mejores resultados fueron obtenidos con la combinación de la inflorescencia, hojas y tallos de C. ambrosioides, a una concentración de $2 \%(\mathrm{p} / \mathrm{p})$ (Cuadro 1). En el caso de los polvos de la inflorescencia de C. ambrosioides, a esta concentración, fueron obtenidos valores de $69,45 \%$, mientras que con polvos de la combinación de hojas y tallos de la misma especie se obtuvo un valor de 67,9\% de mortalidad. Estos datos corresponden a lo señalado por Jacobson (1990) y Rodríguez \& Lagunes (1992), quienes sostienen que esta especie posee propiedades insecticidas, aunque no mencionan valores exactos. También son similares a los valores obtenidos por Tavares (2002), quien al utilizar los polvos de la planta entera de C. ambrosioides (sin las raíces), a una concentración de 1,25\% (p/p), obtuvo una mortalidad de $66,3 \%$ sobre $S$. zeamais; y similares a los obtenidos por Orrego (2004), quien al utilizar los polvos de la misma planta, a una concentración de $1 \%$ (p/p), obtuvo una mortalidad de adultos de $S$. zeamais de $65,8 \%$.

Además, valores parecidos fueron obtenidos por Rodríguez (2000), quien usó el polvo de esta planta a una concentración de $1 \%$ y $3 \%$ (p/p) y obtuvo una mortalidad de S. zeamais de 77,5\% y 100\%, respectivamente. Asimismo, Procópio et al. (2003a) y Silva et al. (2003) lograron un $100 \%$ de mortalidad de S. zeamais con los polvos de esta especie al 1\% y 3\% (p/p), respectivamente. Esto también refrenda los resultados mostrados por C. ambrosioides sobre otras plagas de granos almacenados, como lo señalan Mazzonetto (2002) y Procópio et al. (2003b), quienes obtuvieron un 100\% de mortalidad de Zabrotes subfasciatus y Acanthoscelides obtectus Say, al aplicar polvo de C. ambrosioides a una concentración de 3\% (p/p).

Cuadro 1. Mortalidad y emergencia de adultos de Sitophilus zeamais y pérdida de peso de los granos tratados con los polvos de plantas del género Chenopodium ${ }^{(1)}$.

\begin{tabular}{|c|c|c|c|c|}
\hline Variable & Tratamiento & $0,5 \%$ & $1 \%$ & $2 \%$ \\
\hline \multirow{8}{*}{ Mortalidad } & C. ambrosioides ${ }^{(2)}$ & $37,18 \mathrm{aA}$ & $38,51 \mathrm{aA}$ & $67,90 \mathrm{aA}$ \\
\hline & C. ambrosioides ${ }^{(3)}$ & $37,50 \mathrm{aB}$ & $45,84 \mathrm{aB}$ & $69,45 \mathrm{aA}$ \\
\hline & C. album $^{(2)}$ & $0,00 \mathrm{bB}$ & $6,87 \mathrm{bA}$ & $10,96 \mathrm{bA}$ \\
\hline & C. album $^{(2)}$ & $2,78 \mathrm{bA}$ & $2,08 \mathrm{bA}$ & $4,17 \mathrm{bA}$ \\
\hline & C. quinoa cv. Baer ${ }^{(4)}$ & $4,86 \mathrm{bA}$ & $2,55 \mathrm{bA}$ & $0,62 \mathrm{bA}$ \\
\hline & C. quinoa cv. Faro ${ }^{(4)}$ & $6,02 \mathrm{bA}$ & $1,93 \mathrm{bA}$ & $4,17 \mathrm{bA}$ \\
\hline & C. quinoa cv. Pichaman ${ }^{(4)}$ & $2,00 \mathrm{bA}$ & $6,79 \mathrm{bA}$ & $6,25 \mathrm{bA}$ \\
\hline & Testigo & $0,00 \mathrm{bA}$ & $0,00 \mathrm{bA}$ & $0,00 \mathrm{bA}$ \\
\hline \multirow[t]{8}{*}{ Emergencia } & C. ambrosioides ${ }^{(2)}$ & $41,76 \mathrm{abA}$ & $22,79 b c A B$ & $11,68 \mathrm{cB}$ \\
\hline & C. ambrosioides ${ }^{(3)}$ & $15,72 \mathrm{bA}$ & $7,21 \mathrm{cA}$ & $14,26 \mathrm{bcA}$ \\
\hline & C. album $^{(2)}$ & $84,43 \mathrm{aA}$ & $64,12 \mathrm{abA}$ & $65,06 \mathrm{abcA}$ \\
\hline & C. album $^{(2)}$ & $52,12 \mathrm{abA}$ & $53,59 \mathrm{abA}$ & $68,40 \mathrm{abcA}$ \\
\hline & C. quinoa cv. Baer ${ }^{(4)}$ & $69,18 \mathrm{aA}$ & $73,08 \mathrm{aA}$ & $74,83 \mathrm{abA}$ \\
\hline & C. quinoa cv. Faro ${ }^{(4)}$ & $84,25 \mathrm{aA}$ & $78,27 \mathrm{aA}$ & $78,74 \mathrm{aA}$ \\
\hline & C. quinoa cv. Pichaman ${ }^{(4)}$ & $83,76 \mathrm{aA}$ & $77,66 \mathrm{aA}$ & $82,01 \mathrm{aA}$ \\
\hline & Testigo & $100,00 \mathrm{aA}$ & $100,00 \mathrm{aA}$ & $100,00 \mathrm{aA}$ \\
\hline \multirow[t]{8}{*}{ Pérdida de peso } & C. ambrosioides ${ }^{(2)}$ & $3,05 \mathrm{abA}$ & $2,87 \mathrm{bcAB}$ & $2,47 \mathrm{cB}$ \\
\hline & C. ambrosioides ${ }^{(3)}$ & $2,83 \mathrm{bA}$ & $2,34 \mathrm{cA}$ & $2,30 \mathrm{bcA}$ \\
\hline & C. album $^{(2)}$ & $5,79 \mathrm{aA}$ & $5,93 \mathrm{abA}$ & $5,63 \mathrm{abcA}$ \\
\hline & C. album $^{(2)}$ & $5,88 \mathrm{abA}$ & $5,10 \mathrm{abA}$ & $6,19 \mathrm{abcA}$ \\
\hline & C. quinoa cv. Baer ${ }^{(4)}$ & $6,03 \mathrm{aA}$ & $5,67 \mathrm{aA}$ & $6,20 \mathrm{abA}$ \\
\hline & C. quinoa cv. Faro ${ }^{(4)}$ & $6,44 \mathrm{aA}$ & $5,87 \mathrm{aA}$ & $6,21 \mathrm{aA}$ \\
\hline & C. quinoa cv. Pichaman ${ }^{(4)}$ & $5,74 \mathrm{aA}$ & $6,27 \mathrm{aA}$ & $6,60 \mathrm{aA}$ \\
\hline & Testigo & $6,84 \mathrm{aA}$ & $6,84 \mathrm{aA}$ & $6,84 \mathrm{aA}$ \\
\hline
\end{tabular}

(1)Letras mayúsculas distintas, entre las filas, y letras minúsculas distintas, entre las columnas, indican diferencia significativa entre los tratamientos y concentraciones evaluadas, respectivamente, por el test de Tukey $(\alpha=0,05)$. ${ }^{(2)}$ Hojas y tallos. ${ }^{(3)}$ Inflorescencias. ${ }^{(4)}$ Tallos e inflorescencias. 
En relación a la disminución de la emergencia de adultos los tratamientos se consideraron como promisorios si redujeron, por lo menos en un $50 \%$, la $\mathrm{F}_{1}$ de los insectos (Silva et al., 2003). Los mismos tratamientos que presentaron la mayor mortalidad, fueron también los que presentaron la menor emergencia, destacándose además el tratamiento con los polvos de la inflorescencia de C. ambrosioides al 1\% (p/p), el cual presentó el menor valor de emergencia $(7,21 \%)$ y que no difiere estadísticamente de los otros dos que mostraron mayor mortalidad.

Además, debe destacarse que la interacción entre las concentraciones con los diferentes tratamientos que mostraron la mayor mortalidad no difiere significativamente en la emergencia, haciendo iguales las tres concentraciones (Cuadro 1). Estos resultados concuerdan con lo obtenido por Orrego (2004), quien utilizó polvos de C. ambrosioides al $1 \%(\mathrm{p} / \mathrm{p})$ y obtuvo un $11,6 \%$ de emergencia de adultos de $S$. zeamais. Estos valores también son similares a los reportados por Tavares (2002), quien obtuvo una emergencia de adultos de $S$. zeamais de $28,01 \%$, al utilizar los polvos de C. ambrosioides, a una concentración de 1,25\% (p/p). El 0\% de emergencia de S. zeamais, obtenido por Silva et al. (2003), por Procópio et al. (2003a, 2003b) sobre Z. subfasciatus y A. obtectus, con polvos de C. ambrosioides a las concentraciones de $1 \%$ y $3 \%(\mathrm{p} / \mathrm{p})$ respectivamente, muestra un efecto de los tratamientos superior a lo obtenido en esta investigación, pero sin duda exhiben la misma tendencia de resultados.

Los otros tratamientos, que presentaron una emergencia de adultos inferior al 50\%, fueron las concentraciones de $0,5 \%$ y $1 \%(\mathrm{p} / \mathrm{p})$ de polvo de C. ambrosioides, tanto de inflorescencia como de la mezcla de hojas y tallos. El hecho de que los polvos de la inflorescencia de C. ambrosioides al 1\% (p/p) hayan presentado la menor emergencia, revela que, aún a dosis bajas de polvo de inflorescencia de C. ambrosioides, se pueden obtener valores de emergencia cercanos a cero.

Los tratamientos con menor pérdida de peso fueron aquellos con polvos de C. ambrosioides, sin presentar diferencias significativas en todas sus concentraciones. Las menores pérdidas de peso fueron obtenidas en los polvos de la inflorescencia de C. ambrosioides al $1 \%$ y $2 \%$ (p/p) con valores de 2,34\% y 2,30\% respectivamente, y con la mezcla de hojas y tallos de la misma planta al $2 \%(\mathrm{p} / \mathrm{p})$ con un valor de 2,47\% (Cuadro 1). Esto es similar a lo obtenido por Silva et al. (2003) y por Orrego (2004) con polvos de C. ambrosioides al $1 \%(\mathrm{p} / \mathrm{p})$, que presentaron una pérdida de peso de $0,2 \%$ y de $6,3 \%$, respectivamente, estadísticamente diferentes al testigo en ambos casos.

La razón de que en los casos de mortalidad y emergencia de adultos, los polvos de la inflorescencia de C. ambrosioides presenten mejores resultados que los polvos de la mezcla de las hojas y tallos, se debe a que una mayor concentración de los semioquímicos que tendrían un efecto insecticida y insectistático se encuentra en estos órganos, comprobando lo observado por Tavares (2002), quien obtuvo una diferencia significativa entre los polvos de C. ambrosioides proveniente de sus frutos y los provenientes de la planta entera.

En la prueba de repelencia se evaluó una mezcla de los polvos de las inflorescencias y tallos de C. ambrosioides, debido principalmente a que en la época en que se realizó la recolección, no había plantas con follaje abundante. Los tratamientos evaluados y sus respectivos resultados son señalados en el Cuadro 2. En este cuadro se puede apreciar claramente que todos poseen propiedades repelentes. Los resultados difieren de lo obtenido por Tavares (2002) y por Procópio et al. (2003a), quienes determinaron que los polvos C. ambrosioides no eran repelentes sobre S. zeamais, a una concentración de 3\% (p/p). En el caso de Tavares (2002), el hecho de que los polvos de C. ambrosioides no hayan sido repelentes sobre $S$. zeamais podría explicarse por las estructuras vegetales utilizadas por ese autor, que difieren de las utilizadas en este bioensayo. Aunque, estos resultados sí muestran un comportamiento similar a los obtenidos por Mazzonetto (2002), quien utilizó el polvo de esta misma especie vegetal sobre A. obtectus y Z. subfasciatus, a una concentración de $3 \%(\mathrm{p} / \mathrm{p})$, habiendo determinado que es repelente para estas dos plagas; y por Procópio et al. (2003b), quienes determinaron que polvos de C. ambrosioides, a una

Cuadro 2. Porcentaje de adultos de Sitophilus zeamais atraídos e indice de repelencia de los polvos de la planta completa (hojas y tallos) de Chenopodium ambrosioides ${ }^{(1)}$.

\begin{tabular}{lccl}
\hline Tratamiento & $\begin{array}{c}\text { Concentración } \\
(\%)\end{array}$ & $\begin{array}{c}\text { Insectos atraídos } \\
(\%)\end{array}$ & Indice de repelencia \\
\hline C. ambrosioides & 1 & $30,2 \mathrm{a}$ & 0,604 (repelente) \\
Testigo & - & $69,8 \mathrm{~b}$ & \\
C. ambrosioides & 2 & $37,1 \mathrm{a}$ & 0,742 (repelente) \\
Testigo & - & $62,9 \mathrm{~b}$ & \\
\hline CV $(\%)$ & & 6,58 & \\
\hline
\end{tabular}

(1) Tratamientos con igual letra, en la columna, no difieren estadísticamente por el test de Tukey $(\alpha=0,05)$. 
concentración de 3\% (p/p), son repelentes para Z. subfasciatus.

Para el efecto ovicida y larvicida, como se observa en el Cuadro 3, la emergencia en todos los tratamientos fue de $0 \%$, diferente del testigo que tuvo un total de 88 insectos emergidos. Esto demostraría que la muerte de los huevos y larvas presentes en los granos fue total.

En el efecto fumigante se evaluaron sólo los polvos de C. ambrosioides, por ser la especie más prometedora, utilizándose en esta oportunidad los polvos de sus inflorescencias, hojas y tallos juntos, a las concentraciones de 1\% y 2\% (p/p) (Cuadro 4). En todos los tratamientos se alcanzó un $100 \%$ de mortalidad. Estos resultados superan a los obtenidos por Tavares (2002), quien utilizó los polvos de C. ambrosioides a una concentración de 1,25\% (p/p) y obtuvo una mortalidad de adultos de $78,44 \%$.

En los bioensayos de residualidad sólo se utilizaron los polvos de la inflorescencia de C. ambrosioides al $1 \%$ y $2 \%(\mathrm{p} / \mathrm{p})$, más los polvos de la mezcla de las hojas y tallos de la misma planta al $2 \%(\mathrm{p} / \mathrm{p})$. El análisis de tipo factorial de los datos muestra claramente una interacción entre el tratamiento y el tiempo, y es significativamente mejor el desempeño de la inflorescencia de C. ambrosioides al 2\% (p/p), para las tres variables evaluadas en los cuatro períodos de tiempo.

El porcentaje de mortalidad fue de $100 \%$ en todos los tratamientos, como se observa en el Cuadro 5, a las 24 horas de haber aplicado los polvos, claramente diferentes estadísticamente del testigo. Esto concuerda con lo obtenido por Orrego (2004), quien utilizó polvos de C. ambrosioides a las concentraciones $1 \%$ y $2 \%(\mathrm{p} / \mathrm{p})$ y obtuvo, en el mismo período, una mortalidad de $85,2 \%$ y $100 \%$, respectivamente.

A los 15 días de la aplicación, el polvo de la inflorescencia de C. ambrosioides al 2\% (p/p) mantuvo su eficiencia con un 98,3\% de mortalidad, seguido por el mismo tratamiento, pero a una concentración de $1 \%$ (p/p), con una mortalidad de 53,58\%, siendo estos

Cuadro 3. Emergencia de adultos de Sitophilus zeamais en los bioensayos, para evaluar el efecto ovicida/larvicida de polvos de Chenopodium ambrosioides ${ }^{(1)}$.

\begin{tabular}{llccc}
\hline Especie & $\begin{array}{c}\text { Estructura } \\
\text { vegetal }\end{array}$ & $\begin{array}{c}\text { Concentración } \\
(\%)\end{array}$ & $\begin{array}{c}\mathrm{N}^{\mathrm{o}} \text { de insectos } \\
\text { emergidos }\end{array}$ & $\begin{array}{c}\text { Emergencia } \\
(\%)\end{array}$ \\
\hline C. ambrosioides & Inflorescencias & 1 & 0 & $0,0 \mathrm{a}$ \\
C. ambrosioides & Inflorescencias & 2 & 0 & $0,0 \mathrm{a}$ \\
C. ambrosioides & Hojas y tallos & 2 & 0 & $0,0 \mathrm{a}$ \\
Testigo & & & 88 & $100,0 \mathrm{~b}$ \\
\hline
\end{tabular}

${ }^{(1)}$ Tratamientos con igual letra, en la columna, no difieren estadísticamente por el test de Tukey $(\alpha=0,05)$. tratamientos diferentes estadísticamente entre sí y del testigo.

A los 30 días de la aplicación, sólo el polvo de la inflorescencia de C. ambrosioides al 2\% (p/p) presentó una mortalidad sobre el 50\% (54,85\%), mientras que los demás tratamientos presentaron un valor inferior al 15\% y fueron iguales estadísticamente entre sí y al testigo. A los 45 días de la aplicación, todos los tratamientos fueron iguales estadísticamente al testigo y el porcentaje de mortalidad fue menor al 35\%. En consecuencia, basándose en esto, se puede inferir que la vida útil de los polvos de C. ambrosioides, como controlador efectivo de $S$. zeamais, no supera los 15 días, por lo cual se deberían hacer aplicaciones sucesivas de polvo recién secado y molido, si se quiere almacenar algún tipo de grano por períodos de tiempo mayores que éste. Además, esta evaluación demostraría que es en la inflorescencia donde se encuentra la mayor concentración de compuestos con efecto insecticida, ya que fue este tratamiento el que más perduró en el tiempo con una alta mortalidad.

En relación a la $\mathrm{F}_{1}$, el bioensayo realizado 24 horas después de la aplicación de los polvos, la emergencia de insectos adultos fue en todos los tratamientos de $0 \%$, siendo claramente diferente estadísticamente del testigo (Cuadro 5), lo que es similar a lo obtenido por Orrego (2004), quien en el bioensayo de las 24 horas obtuvo un $10,8 \%$ y $3,6 \%$ de emergencia, utilizando polvo de C. ambrosioides al $1 \%$ y $2 \%(\mathrm{p} / \mathrm{p})$, respectivamente.

A los 15 días, sólo el tratamiento de polvos de la inflorescencia de C. ambrosioides al 2\% (p/p) continuó con una emergencia igual a $0 \%$, aunque todos los tratamientos continuaron siendo iguales estadísticamente entre sí y distintos del testigo.

A los 30 días se observa que la emergencia de adultos comienza a ser mayor y que ya no se muestra tan fuertemente reducida como en los casos anteriores, presentándose la menor emergencia nuevamente con

Cuadro 4. Mortalidad de adultos de Sitophilus zeamais en los bioensayos, para evaluar el efecto fumigante de los polvos de Chenopodium ambrosioides ${ }^{(1)}$.

\begin{tabular}{lcc}
\hline Especie & Concentración (\%) & Mortalidad (\%) \\
\hline C. ambrosioides & 1 & $100,0 \mathrm{a}$ \\
C. ambrosioides & 2 & $100,0 \mathrm{a}$ \\
C. ambrosioides & 2 & $100,0 \mathrm{a}$ \\
Testigo & & $0,0 \mathrm{~b}$ \\
\hline
\end{tabular}

${ }^{(1)}$ Tratamientos con igual letra, en la columna, no difieren estadísticamente por el test de Tukey $(\alpha=0,05)$. 
Cuadro 5. Mortalidad, emergencia de adultos y pérdida de peso de granos, en los bioensayos de residualidad con polvos de C. ambrosioides $^{(1)}$.

\begin{tabular}{|c|c|c|c|c|c|}
\hline \multirow[t]{2}{*}{ Variable } & \multirow[t]{2}{*}{ Tratamiento } & \multicolumn{4}{|c|}{ IDDA $^{(2)}$} \\
\hline & & 1 & 15 & 30 & 45 \\
\hline \multirow[t]{4}{*}{ Mortalidad $^{(3)}$} & Testigo $(0 \%)$ & ObA & $0,00 \mathrm{bA}$ & $0,00 \mathrm{bA}$ & $0,00 \mathrm{aA}$ \\
\hline & Inflorescencia (1\%) & $100 \mathrm{aA}$ & $53,58 \mathrm{abB}$ & $6,36 \mathrm{bD}$ & $17,41 \mathrm{aC}$ \\
\hline & Inflorescencia (2\%) & $100 \mathrm{aA}$ & $98,30 \mathrm{aB}$ & $54,85 \mathrm{aC}$ & $31,39 \mathrm{aD}$ \\
\hline & Hojas y tallos $(2 \%)$ & $100 \mathrm{aA}$ & $32,15 \mathrm{abB}$ & $13,05 \mathrm{bD}$ & $19,17 \mathrm{aC}$ \\
\hline \multirow[t]{4}{*}{ Emergencia $^{(4)}$} & Testigo $(0 \%)$ & $100 \mathrm{aA}$ & $100,00 \mathrm{aA}$ & $100,00 \mathrm{aA}$ & $100,0 \mathrm{aA}$ \\
\hline & Inflorescencia (1\%) & ObD & $24,24 \mathrm{bC}$ & $31,48 \mathrm{bA}$ & $24,3 \mathrm{bB}$ \\
\hline & Inflorescencia $(2 \%)$ & ObC & $0,00 \mathrm{bC}$ & $1,85 \mathrm{bB}$ & $30,3 \mathrm{bA}$ \\
\hline & Hojas y tallos $(2 \%)$ & $0 \mathrm{bC}$ & $9,09 \mathrm{bB}$ & $50,0 \mathrm{abA}$ & $50,0 \mathrm{abA}$ \\
\hline \multirow[t]{4}{*}{ Pérdida de peso ${ }^{(4)}$} & Testigo $(0 \%)$ & $4,35 \mathrm{aA}$ & $2,55 \mathrm{aD}$ & $2,84 \mathrm{aC}$ & $3,14 \mathrm{aB}$ \\
\hline & Inflorescencia (1\%) & $1,49 \mathrm{bB}$ & $1,05 \mathrm{bcC}$ & $1,07 \mathrm{bC}$ & $2,24 \mathrm{aA}$ \\
\hline & Inflorescencia ( $2 \%)$ & $1,48 \mathrm{bB}$ & $1,27 b C$ & $1,29 b C$ & $1,93 \mathrm{aA}$ \\
\hline & Hojas y tallos $(2 \%)$ & $1,57 \mathrm{bB}$ & $0,71 \mathrm{cD}$ & $1,49 \mathrm{bC}$ & $2,00 \mathrm{aA}$ \\
\hline
\end{tabular}

(1)Letras mayúsculas distintas entre las filas, y letras minúsculas distintas, entre las columnas, indican diferencia significativa entre los tratamientos y fechas, respectivamente, por el test de Tukey $(\alpha=0,05) .{ }^{(2) I D D A}$ infestación de los granos, días después de la aplicación de los polvos.

(3)Parámetro evaluado a los 15 días de realizada la infestación. ${ }^{(4)}$ Parámetros evaluados a los 55 días de realizada la infestación.

los polvos de la inflorescencia de C. ambrosioides al $2 \%(\mathrm{p} / \mathrm{p})$, con un valor de $1,85 \%$.

Finalmente, a los 45 días, todos los tratamientos presentaron una emergencia superior al 24\%, habiendo sido estos valores los más altos de la evaluación.

Una baja emergencia se debe principalmente a una alta mortalidad, pero además hay que considerar el efecto ovicida y larvicida de los polvos que, como se observó anteriormente, provocó una alta mortalidad de huevos y larvas. Esto explicaría por lo tanto, el porque la emergencia de los polvos de la inflorescencia de C. ambrosioides al $2 \%$ (p/p), en el bioensayo de 30 días, fue de $1,85 \%$, mientras que la mortalidad fue de sólo un $54,85 \%$ para el mismo período de tiempo. Como se observa en el Cuadro 5, en todos los tratamientos y en todas las evaluaciones, la pérdida de peso de estos difiere del testigo, a excepción del bioensayo 45 días después de la aplicación de los polvos, donde los tratamientos y el testigo fueron iguales estadísticamente.

Aunque, en general la pérdida de peso de los granos fue inferior al 3,2\%. Al mostrar esta última variable una menor relevancia en el comportamiento de los polvos vegetales, en el tiempo se realizó una regresión solo para mortalidad y emergencia versus el tiempo. Estos estudios muestran claramente que en la medida que el tiempo aumenta, existe una relación inversa para la mortalidad y directa para la emergencia de insectos (Figura 1). Los ajustes obtenidos y los valores para los respectivos coeficientes de correlación varían entre un 0,69 y 0,9, para la mortalidad, y entre 0,54 y 0,86, para la emergencia,
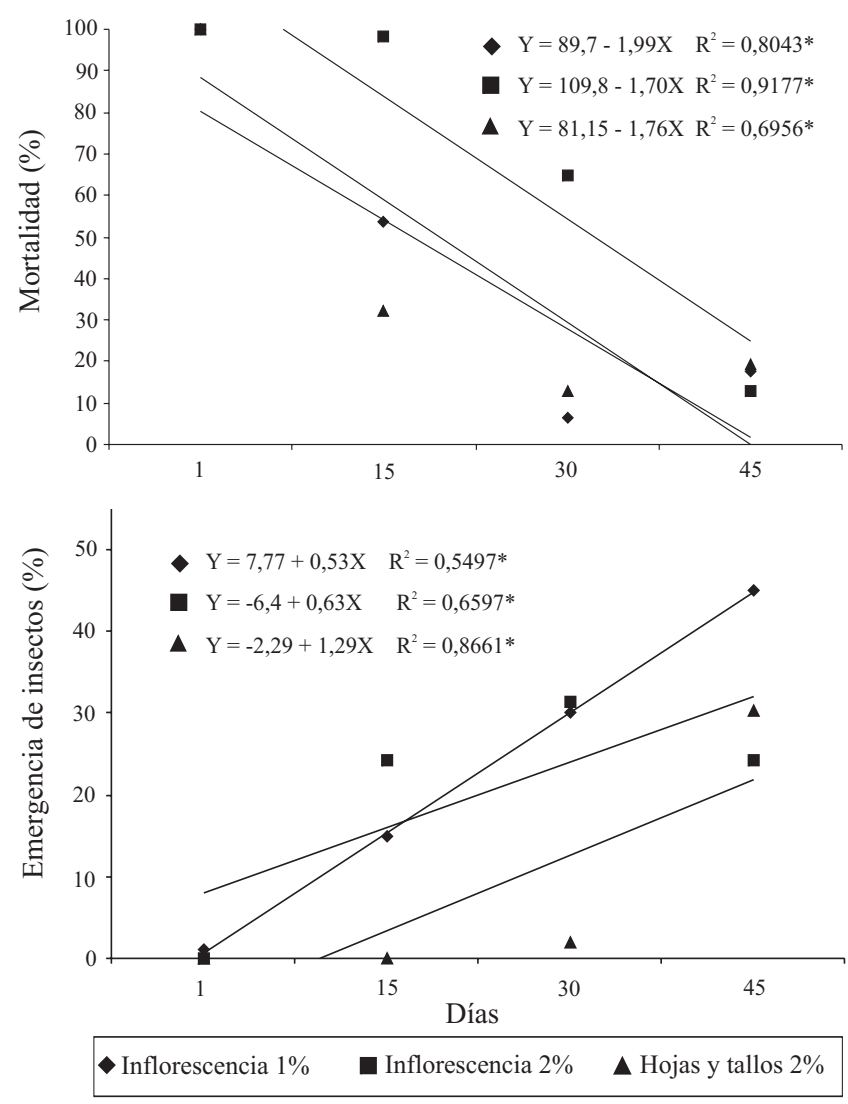

Figura 1. Porcentaje de mortalidade y de emergencia de Sitophilus zeamais Motschulsky, a los 1, 15, 30 y 45 días, en maíz cultivar Laredo tratado con polvo de Chenopodium ambrosioides.

lo que confirma la gran variabilidad que poseen, en general, las sustancias insecticidas de origen vegetal y que, entre otras cosas, han impedido su masificación. 
Finalmente, se puede decir que todos los resultados obtenidos en el presente trabajo son de gran utilidad, para considerar a los polvos de C. ambrosioides como una alternativa real para el control de $S$. zeamais y factible de llevar a cabo en la práctica, en especial por pequeños agricultores.

\section{Conclusiones}

1. De las tres especies del género Chenopodium evaluadas, solamente el polvo de Chenopodium ambrosioides L. controla eficazmente Sitophilus zeamais Motschulsky en maíz almacenado.

2. La inflorescencia de Chenopodium ambrosioides L. presenta las mayores propiedades insecticidas para el control de Sitophilus zeamais Motschulsky.

3. El polvo de Chenopodium ambrosioides presenta efecto ovicida, larvicida, fumigante y repelente.

\section{Referencias}

ABBOTT, W.S. A method for computing the effectiveness of an insecticide. Journal of Economic Entomology, v.18, p.265-267, 1925.

ADAMS, J.M.; SCHULTEN, G.G.M. Losses caused by insects, mites and microorganisms. In: American Association of Cereal Chemists. Postharvest grain loss assessment methods. Slough, England, 1976. p.83-93.

CELIS, J.; KUNADU, K. Pest control by non-chemical methods and reduced levels of chemicals in grain storage: a review. Agro Sur, v.20, p.56-65, 1992.

DELL'ORTO H.; ARIAS, C. Distribución e importancia de los insectos que dañan granos y productos almacenados en Chile. Santiago, Chile: FAO/INIA, 1983. 67p.

GOLOB, P.; HANKS, C. Protection of farm stored maize against infestation by Prostephanus truncatus (Horn) and Sitophilus species in Tanzania. Journal of Stored Product Research, v.26, p.187198, 1990.

HALSTEAD, D.G.H. External sex differences in stored-products coleoptera. Bulletin of Entomological Research, v.54, p.119-134, 1963.

JACOBSON, M. Glossary of plant-derived insect deterrents. Boca Raton, Florida: CRC Press, 1990. 213p.

LAGUNES, A.; RODRÍGUEZ, C. Búsqueda de la tecnología apropiada para el combate de plagas del maíz almacenado en condiciones rústicas. Montecillo, México: CONACYT/Colegio de Postgraduados, 1989. 150p.

LARRAÍN, P. Manejo integrado de plagas en granos almacenados. Investigación y Progreso Agropecuario, v.81, p.10-16, 1994.

MAREGGIANI, G. Manejo de insectos plagas mediante sustancias semioquímicas de origen vegetal. Manejo Integrado de Plagas, v.60, p.22-30, 2001.

MAZZONETTO, F. Efeito de genótipos de feijoeiro e de pós de origem vegetal sobre Zabrotes subfasciatus (Boh.) e Acanthoscelides obtectus (Say) (Col: Bruchidae). 2002. 134p. Tese (Doutorado) - Universidade de São Paulo, Piracicaba.

ORREGO, O. Búsqueda de plantas nativas y ornamentales para el control de Sitophilus zeamais Motschulsky en granos almacenados. 2004. 30p. Tesis (Graduación) - Universidad de Concepción, Chillán, Chile.

PAEZ, A. Uso de polvos vegetales e inertes minerales como una alternativa para el combate del gorgojo del maíz Sitophilus zeamais Motschulsky (Coleoptera: Curculionidae) en maíz almacenado. 1987. 135p. Tesis (Magister) - Colegio de Postgraduados en Ciencias Agrícolas, Montecillo, México.

PROCÓPIO, S.; VENDRAMIM, J.; RIBEIRO, J.; SANTOS, J.B. Bioatividade de diversos pós de origem vegetal em relação a Sitophilus zeamais Mots. (Coleoptera: Curculionidae). Ciência e Agrotecnologia, v.27, p.1231-1236, 2003a.

PROCÓPIO, S.; VENDRAMIM, J.; RIBEIRO, J.; SANTOS, J.B. Efeito de pós vegetais sobre Acanthoscelides obtectus (Say) e Zabrotes subfasciatus (Boh.) (Coleoptera: Bruchidae). Revista Ceres, v.50, p.395-405, 2003b.

RODRÍGUEZ, C. Propiedades plaguicidas del epazote Teloxys ambrosioides (Chenopodiaceae). In: SIMPOSIO NACIONAL SOBRE SUSTANCIAS VEGETALES Y MINERALES EN EL COMBATE DE PLAGAS, 6., 2000. Acapulco. [Anais]. Acapulco: Sociedad Mexicana de Entomología, 2000. p.95-110.

RODRÍGUEZ, C.; LAGUNES, A. Plantas con propiedades insecticidas; resultados de pruebas experimentales en laboratorio, campo y granos almacenados. Agroproductividad, v.1, p.17-25, 1992.

SILVA, G.; LAGUNES, A.; RODRÍGUEZ, J.C. Control de Sitophilus zeamais (Coleoptera: Curculionidae) con polvos vegetales solos y en mezcla con carbonato de calcio en maíz almacenado. Ciencia e Investigación Agraria, v.30, p.153-160, 2003.

SILVA, G.; LAGUNES, A.; RODRÍGUEZ, J.C.; RODRÍGUEZ, D. Insecticidas vegetales; una vieja nueva opción en el manejo de insectos. Manejo Integrado de Plagas y Agroecología, v.66, p.4-12, 2001.

TAVARES, M.A.G.C. Bioatividade da erva-de-santa-maria, Chenopodium ambrosioides L. (Chenopodiaceae), em relação a Sitophilus zeamais (Col.: Curculionidae). 2002. 59p. Tese (Mestrado) - Universidade de São Paulo, Piracicaba.

WEAVER, D.; SUBRAMANYAM, B. Botanicals. In: SUBRAMANYAN, B.; HAGSTRUM, D.W. (Ed.). Alternatives to pesticides in stored-product IPM. Boston: Kluwer Academics, 2000. p.303-320. 